\title{
In Silico Study of Protein-Protein Interactions in Mapks and pp2cs of Medicago sativa Discloses its Docking Sites Variations
}

\section{Muhammad Naveed Shahid ${ }^{1 *}$}

https://orcid.org/0000-0003-4417-7126

\section{Sania Rasheed 1}

https://orcid.org/0000-0002-6470-1450

Adil Jamal ${ }^{2}$

https://orcid.org/0000-0002-8724-5566

\section{Sana Khalid ${ }^{3}$}

https://orcid.org/0000-0001-9051-3528

\section{Zeeshan Shamim ${ }^{4}$}

https://orcid.org/0000-0002-7948-7653

${ }^{1}$ University of Education, Division of Science and Technology, Department of Botany, Lahore, Punjab, Pakistan; ${ }^{2} \mathrm{Umm}$ Al Qura University, Science and Research College of Nursing, Makkah, Kingdom of Saudi Arabia; ${ }^{3}$ Lahore College for Woman University, Department of Botany, Lahore, Punjab, Pakistan; ${ }^{4}$ Mirpur University of Science and Technology, Department of Biotechnology, Mirpur, AJK, Pakistan.

Editor-in-Chief: Alexandre Rasi Aoki

Associate Editor: Marcelo Ricardo Vicari

Received: 2021.03.21; Accepted: 2021.07.02.

*Correspondence: naveed.shahid@ue.edu.pk (M.N.S.).

\section{HIGHLIGHTS}

- Protein-protein interactions (PPIs) provide a basis for understanding the biological and molecular processes within the cell

- $\mathrm{MP2C}$ is regulated in the response of various abiotic-stresses.

- In plants, the most significant signaling mechanism engaged in abiotic stress response is the MAPK cascade, which transforms the environmental and growth signal into an intracellular response.

- For the prediction of accurate and high-quality PPIs, high-throughput in silico techniques are use.

Abstract: A wide variety of cellular mechanisms such as cell division and metabolic processes are maintained by protein-protein interactions (PPIs). The identification of PPI through laboratory techniques is costly, time-consuming, difficult, and challenging. However, computational techniques were generated for PPIs prediction. In alfalfa (Madicago sativa), PPI was predicted among 12 MsMAPKs and 4 MsPP2Cs using a docking approach. For homology modelling, the Swiss model was employed while PROCHECK, ERRAT, 
and Verify3D were used to validate 3D models. The Ramachandran plots were obtained from PROCHECK which showed value more than 90\% (nPP2C1, PP2C1, PP2C, and MSK-3 revealed $92.9 \%, 94.2 \%, 92.4 \%$, and $91.1 \%$ respectively) for high-quality structures. The HawkDock server and the BIPSPI server were used to analyse protein docking and predict interaction sites, respectively. Our findings demonstrated that MsPP2C docking sites play an important role in the identification and docking of MsMAPKs. The binding free energy ranged from $-0.16 \mathrm{Kcal} / \mathrm{mol}$ to $-49.15 \mathrm{Kcal} / \mathrm{mol}$ for all MsMAPKs and MsPP2Cs, indicating that they interact. Docking site analysis showed that there were 48 pairs of PPIs which indicated that MsPP2Cs can perform a vital role in other signaling pathways. This study found that all MsPP2Cs have docking sites for MsMAPKs, indicating that this method can accurately determine protein-protein interactions.

Keywords: Alfalfa; Homology Modeling; MsMAPKs; MsPP2Cs; Molecular Docking.

\section{INTRODUCTION}

To detect plant growth and development, plants have a signal transduction mechanism that activates responses to hormones, nutrients, environmental signals, and stresses [1]. Proteins are phosphorylated and dephosphorylated in plants which are the universal post-translational modifications. In the signaling pathway, phosphorylation of protein is carried out by protein kinases (PKs) and phosphorylation of reversible protein is accomplished by protein phosphatases (PPs). However, many proteins can target by reversible protein phosphorylation along with protein kinases themselves [2].

In plants, the most significant signaling mechanism engaged in abiotic stress response is the MitogenActivated Protein Kinase (MAPK) cascade, which transforms the environmental and growth signal into an intracellular response [3]. The kinases involved in MAPKs are MAPKKKs, MAPKKs, and MAPKs [4]. These MAPKs are necessary for phosphorylation and activation of a MAPK cognate. Therefore, activation of MAPK is phosphorylated by downstream substrates that contain transcriptional regulation [5]. While PP2Cs depend on $\mathrm{Mn}^{+2}$ and $\mathrm{Mg}^{+2}$ ions and these proteins are monomeric enzymes [6]. Protein Phosphatase 2Cs (PP2Cs) act as a negative regulator of MAPKs that activates during plant stresses. However, it is examined that PP2Cs in Medicago sativa proceed in negative regulation during the signaling pathway of MAPK. MP2C is regulated in the response of various stresses for example drought, salinity, and wound in alfalfa [7].

MAPKs are phosphorylated by transcriptional factors and particular activators such as MAPKKs and MKKs which are activated by double phosphorylation on threonine and tyrosine [8]. While PP2Cs cause inactivation of MAPKs by dephosphorylation of serine/threonine and tyrosine residues [9]. Protein-protein interactions (PPIs) involve a wide range of biological processes, such as developmental regulation, metabolic processes, or interactions between cells [10]. Therefore, PPIs provide a basis for understanding the biological and molecular processes within the cell. Those amino acids that take part in stable PPIs induce mutational variations during development. Therefore, interacting proteins have increased the likelihood of genes being encoded that have the same physiology. However, genomic structure evaluation can provide understanding about PPI [11].

Several laboratory methods for example yeast two-hybrid $(\mathrm{Y} 2 \mathrm{H})$ screens and high-throughput biological techniques were performed for the identification of protein interactions [12]. These techniques were costly, difficult, and time-taken and were not helpful for all types of plants [13]. Furthermore, experimental techniques give false-positive outcomes [14] and could be explained as low throughput. These limitations provide a gap for the detection of new PPIs through laboratory experiments [15]. For the prediction of accurate and highquality PPIs, high-throughput in silico techniques are used [16] which can easily detect protein-protein interaction [13]. Therefore, in silico approaches have been proposed to deal with the challenges of the identification of three-dimensional structures and PPIs prediction. In several docking methods, detailed data that produce a broad range of structural modeling and identify the required docking locations with unique characteristics can be identified [17]. Through laboratory techniques, the determination of the complex threedimensional structure of a protein is very challenging [18].

The aim of this study was the use of in silico docking approach for the prediction of PPIs regarding MsPP2Cs and MsMAPKs which show novel docking site variants in $M$. sativa. The in silico technique is to compensate for the difficulty in determining the network of PPIs. This work provides valuable insight into PPIs which includes signal transduction and plays a major function in biological processes. 


\section{MATERIALS AND METHODS}

\section{Retrieval of proteins sequences}

In Medicago sativa L. 4 protein phosphates 2Cs (MsPP2Cs) and 12 Mitogen-activated protein kinases (MsMAPKs) have been discovered up till now. These 4 MsPP2Cs and 12 MsMAPKs were used for proteinprotein interaction study. The sequences of MP2C, NPP2C1, PP2C1 and PP2C with accession numbers O24078, Q4L0F8, A0A4Y5UM19 and A0A0K2K072 respectively and MAPK, MMK1, MMK2, MMK3, MMK4, TDY1, SIMKK, MEKK1, MSK-1, MSK-2, MSK-3 and PRK with accession numbers Q1ZZH6, Q07176, Q40353, Q9ZP91, O24077, Q9XF36, Q93WR8, Q7XTK4, P51137, P51138, P51139 and Q93WR7 respectively were obtained from UniProt database https://www.uniprot.org/.

\section{Homology Modeling}

Homology modeling was performed with the help of the Swiss model. In comparative modeling, 3-D structures of target sequences were built and experimental evidence relating to evolutionary knowledge on protein structure was used as a template.

\section{Analysis of three-dimensional models}

The analysis and visualization of three-dimensional models of proteins were performed by UCSF Chimera (https://www.cgl.ucsf.edu/chimera/) and PyMOL (https://pymol.org/2/).

\section{Refinement, Evaluation, and Validation of the $3 \mathrm{~d}$ Models}

3Drefine was used for the refinement of all MsMAPKs and MsPP2Cs. PROCHECK, ERRAT, and VERIFY 3D was used to evaluate and validate 3D models.

\section{Docking Studies}

For prediction and analysis of PPIs structures, the HawkDock server [19] was performed by applying the docking algorithm 'ATTRACT' [20]. MM/GBSA [21] was performed for the role of scoring and analysis of the free energy decomposition. MsMAPKs 3D structures were deposit as receptors and MsPP2Cs as ligands in form of PDB as input. To predict the complex structures of PPIs, the ATTRACT algorithm was used. For the prediction of key residues, MM/GBSA was used and this is relay on force field (ffo2) and GBOBC1 which provided the greatest binding energy prediction. The scores and energy in the lowest value lead to valuable prediction in the HawkDock server [22].

\section{Selection of Scores/Binding Energies as Criterion}

A valuation criterion was selected to distinguish the interacting and non-interacting residues among MsMAPKs and MsPP2Cs. It has been previously clarified for docking sites that MAPK phosphatase interaction was only found in subgroup B protein phosphatases (PP2C) with different types of MPKs which have been shown by experimental techniques [23]. The binding energies of the interacting proteins were examined on basis of the concept that homologous genes play an identical function.

\section{PPIs sites prediction}

xgBoost interface prediction of specific partner interaction (BIPSPI) (http://bipspi.cnb.csic.es/xgbPredApp/) server was used for the prediction of PPIs sites. The precision threshold was set (0.450) for MsMAPKs (receptors) and MsPP2Cs (ligands).

\section{Interolog Based PPIs prediction and Comparison of MAPKs Docking Sites}

To analyze the protein-protein interaction among MsMAPKs and MsPP2Cs, 3D structures of proteins were used. The comparison was performed between Simkk, AtMMK2, DMEK, AtMMK5, AtMMK4, mek3, and mek1 of plants, yeast, and human. 


\section{RESULTS}

\section{Model Building}

In alfalfa, 12 MsMAPKs and 4 MsPP2Cs were analyzed and 3D models of these proteins were obtained by using Swiss model. All MsPP2Cs and MsMAPKs results of the Swiss model in the form of GMQE, oligostate, and QMEAN Z-score were obtained. However, the Swiss model relay on ProMod3 [24]. UCSF chimera was used for visualization of 4 MsPP2Cs and 12 MsMAPKs models. The residues in the hydrophobic region are indicated in yellow colour and residues having a negative charge in green (Figure 1).

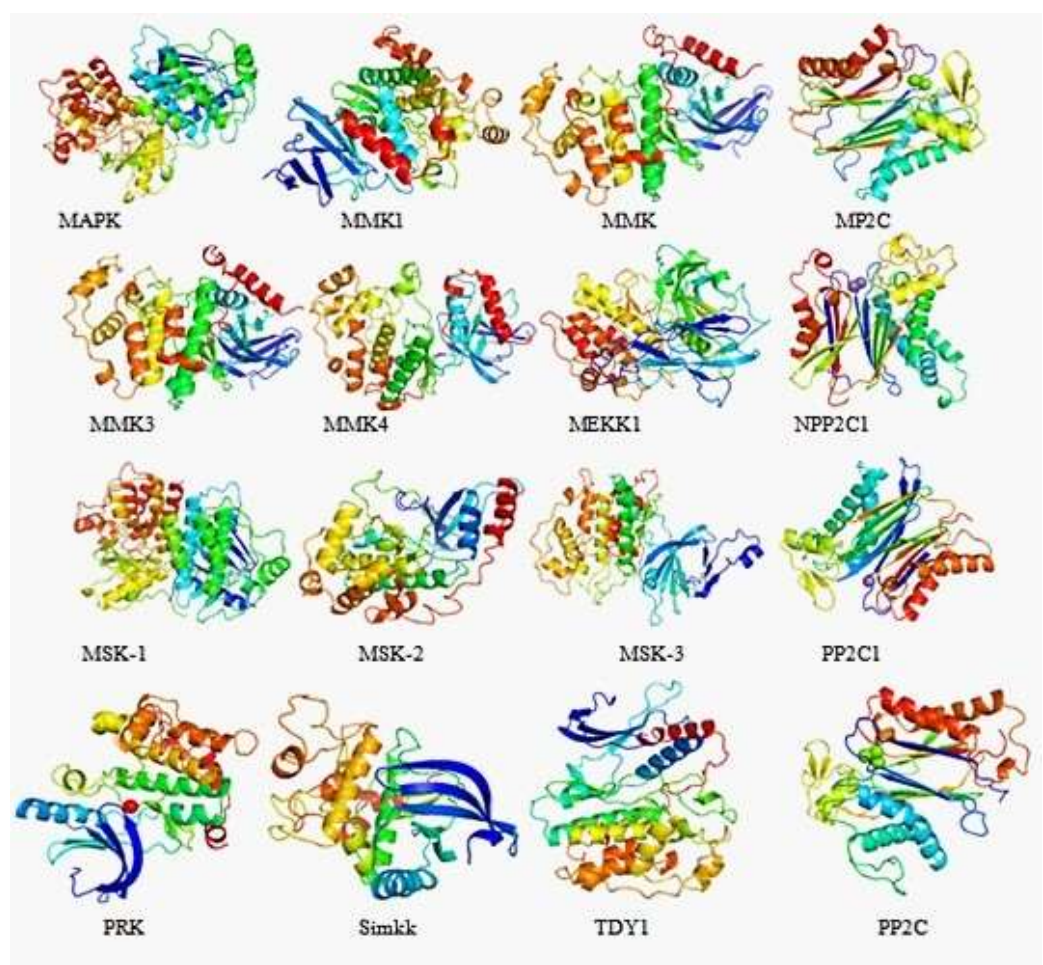

Figure 1. 3-D models of MsMAPKs and MsPP2Cs obtained through 'Swiss model' and visualized by UCSF chimera. The yellow colour indicated residues in the hydrophobic region and negatively charged in green.

\section{3-D model Refinement, Evaluation, and Validation}

3D refine tool was used for refinement of three-dimensional models of 12 MsMAPKs and 4 MsPP2Cs which revealed score, Mol Probity, and RW Plus. The 3D refine scores for MMK1, MMK2, MMK3, MMK4, TDY1, MAPK, PRK, SIMKK, MEKK1, MSK-1, MAK-2 and MSK-3 were 22541.4, 23464.3, 22398.0, 23470.2, 23988.2, 22145.8, 17330.0, 19858.4, 29913.6, 24621.8, 23516.1, 22161.5 respectively and for NPP2C1, PP2C1, PP2C and MP2C were 19143.3, 14040.3, 14652.0 and 14245.2 respectively. Validation of refined models was checked through Ramachandran plots in the 'PROCHECK' tool which indicated the amino acid percentage of both MsMAPKs and MsPP2Cs. According to PROCHECK [25] the percentage of residues in most favored region should be $90 \%$ for good quality model and better stereo-chemical property in the core region of Ramachandran plots. These Ramachandran plots showed the most favored, additional allowed, generously allowed, and disallowed region residue in red, yellow, light yellow, and white respectively. The percentages of residues in most favored region for NPP2C1, PP2C1, PP2C and MP2C were shown 92.9\%, 94.2\%, 92.4\% and 91.1\% respectively and for MMK1, MMK2, MMK3, MMK4, TDY1, MAPK, PRK, SIMKK, MEKK1, MSK-1, MAK-2 and MSK-3 were 90.2\%, 94.5\%, 90.8\%, 91.1\%, 96.5\%, 93.7\%, 92.5\%, 90.3, 94.4\%, $90.1 \%, 95.5 \%$ and $91.1 \%$ respectively. The ERRAT and Verify 3D was used for further study and differentiate the regions that are correctly and incorrectly generated that depend on features of atomic interactions [26]. The overall quality factor in ERRAT analysis for MP2C, nPP2C1, PP2C1 and PP2C was 91.566, 91.054, 91.154 and 94.224) and for MMK1, MMK2, MMK3, MMK4, TDY1, MAPK, PRK, simkk, mekk1, MSK-1, MAK2 and MSK-3 was 93.660, 90.616, 94.169, 90.173, 92.121, 96.428, 86.940, 85.062, 79.587, 96.429, 91.290 and 94.986 respectively. In VERIFY 3D, residues with an average 3D/1D score were maximum in MAPK and MSK-1 that was $97.03 \%$ and the graph values of MsMAPKs and MsPP2Cs models were 0.6 to 0.8. 


\section{Docking Study of MsMAPKs and MsPP2Cs}

To determine the interaction between subgroups of MsMAPK and MsPP2Cs, protein-protein docking was performed. PP2C has an enzyme that acts as a ligand for MAPK and relay on $\mathrm{Mg}^{2+} / \mathrm{Mn}^{2+}$ [27]. The models of MsPP2Cs showed $\mathrm{Mg}^{2+} / \mathrm{Mn}^{2+}$ ions and their connection with residues. For the docking study, MsPP2Cs were kept as the ligand, and MsMAPKs were taken as a receptor which revealed the best and lowest score in HawkDock and MM-GBSA study. The lowest energy was obtained in MP2C-MSK2 (-49.15 $\mathrm{Kcal} / \mathrm{mol}$ ) and the lowest score was shown in MP2C-simkk (-7341.86) (Table 1). The stable models and highest binding interactions were demonstrated by low (negative) energy and the low score value. The energy range was -0.16 to -49.15 and the score range was -4788.55 to -7341.86 for all docking pairs. The results of docking were visualized through pymol (Figure 2). The predicted results in this study indicated the highest interaction between binding proteins. The MsPP2Cs i.e. PP2C, PP2C1, MP2C, and nPP2C1 were highlighted in limon, cyan, green, and yellow-orange color respectively, and MsMAPKs i.e. MAPK, MMK1, MSK-1, MSK2, MSK-3, TDY1, Simkk, MMK2, MMK3, MMK4, mekk1, and PRK were in violet, multi-color, grey, pink, light blue, purple, light pink and cyan color respectively.

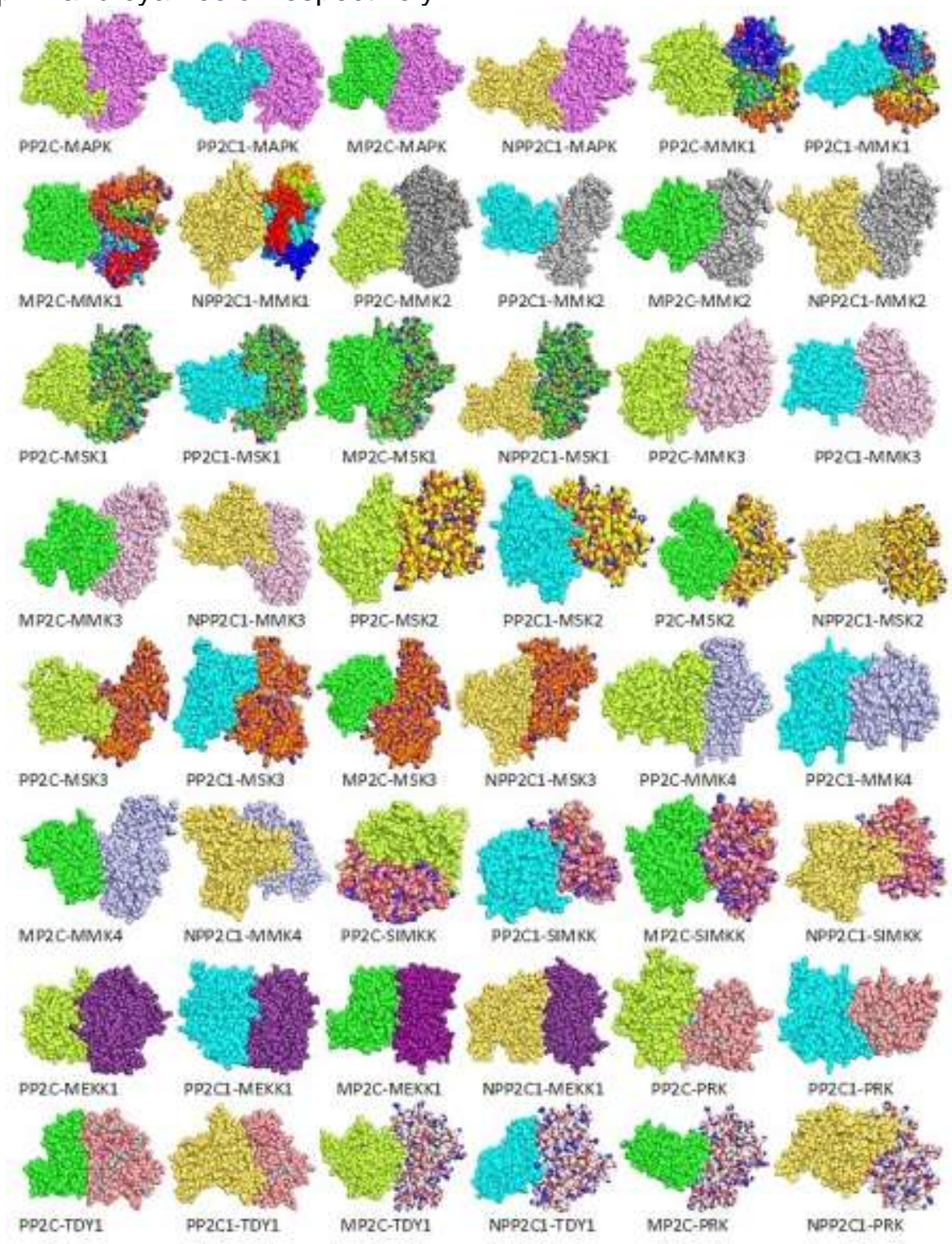

Figure 2. Docking of $M$. sativa PP2Cs and MAPKs. MSPP2Cs (PP2C, PP2C1, MP2C, and nPP2C1) are shown in limon, cyan, green and yellow-orange color respectively. While MsMAPKs (MAPK, MMK1, MSK-1, MSK-2, MSK-3, TDY1, Simkk, MMK2, MMK3, MMK4, mekk1, and PRK) shown in violet, multi-color, grey, pink, light blue, purple, light pink, and cyan color respectively. 
Table 1. Docking scores and binding energies of MsMAPKs and MsPP2Cs obtained from HawkDock server.

\begin{tabular}{|c|c|c|}
\hline PPIs & Score & Binding Energy of Complex $(\mathrm{Kcal} / \mathrm{mol})$ \\
\hline MP2C-MAPK & -5573.51 & -9.53 \\
\hline MP2C-MEKK1 & -6632.78 & -14.22 \\
\hline MP2C-MMK1 & -6000.49 & -43.63 \\
\hline MP2C-MMK2 & -6603.67 & -33.64 \\
\hline MP2C-MMK3 & -5814.63 & -8.65 \\
\hline MP2C-MMK4 & -6535.95 & -39.49 \\
\hline MP2C-TDY1 & -6075.63 & -23.46 \\
\hline MP2C-SIMKK & -7341.86 & -42.59 \\
\hline MP2C-MSK1 & -5573.51 & -10.06 \\
\hline MP2C-MSK2 & -6024.78 & -49.15 \\
\hline MP2C-MSK3 & -5749.20 & -8.31 \\
\hline MP2C-PRK & -5807.86 & -3.37 \\
\hline PP2C-MAPK & -5617.76 & -4.99 \\
\hline PP2C-MEKK1 & -5217.63 & -5.6 \\
\hline PP2C-MMK1 & -5147.52 & -0.16 \\
\hline PP2C-MMK2 & -5521.08 & -8.95 \\
\hline PP2C-MMK3 & -4892.35 & -20.27 \\
\hline PP2C-MMK4 & -4788.55 & -24.73 \\
\hline PP2C-TDY1 & -5832.47 & -18.04 \\
\hline PP2C-SIMKK & -6409.73 & -36.37 \\
\hline PP2C-MSK1 & -5617.76 & -0.72 \\
\hline PP2C-MSK2 & -4834.07 & -12.22 \\
\hline PP2C-MSK3 & -5033.79 & -32.32 \\
\hline PP2C-PRK & -5434.70 & -18.26 \\
\hline PP2C1-MAPK & -5845.77 & -7.53 \\
\hline PP2C1-MEKK1 & -6982.31 & -19.66 \\
\hline PP2C1-MMK1 & -6160.70 & -22.89 \\
\hline PP2C1-MMK2 & -5569.71 & -27.26 \\
\hline PP2C1-MMK3 & -5921.18 & -35.98 \\
\hline PP2C1-MMK4 & -5427.77 & -11.7 \\
\hline PP2C1-TDY1 & -6374.08 & -22.22 \\
\hline PP2C1-SIMKK & -5856.44 & -34.99 \\
\hline PP2C1-MSK1 & -5845.77 & -9.67 \\
\hline PP2C1-MSK2 & -5562.28 & -35.27 \\
\hline PP2C1-MSK3 & -5860.94 & -31.15 \\
\hline PP2C1-PRK & -4934.05 & -18.29 \\
\hline NPP2C1-MAPK & -5532.41 & -11.41 \\
\hline NPP2C1-MEKK1 & -6318.35 & -10.22 \\
\hline NPP2C1-MMK1 & -5872.84 & -19.46 \\
\hline NPP2C1-MMK2 & -6040.60 & -25.86 \\
\hline NPP2C1-MMK3 & 6125.20 & -22.49 \\
\hline NPP2C1-MMK4 & -6211.05 & -18.42 \\
\hline NPP2C1-TDY1 & -5783.76 & -4.84 \\
\hline NPP2C1-SIMKK & -5869.84 & -20.86 \\
\hline NPP2C1-MSK1 & -5532.41 & -13.86 \\
\hline NPP2C1-MSK2 & -5215.38 & -36.02 \\
\hline NPP2C1-MSK3 & -6549.31 & -34.25 \\
\hline NPP2C1-PRK & -6188.98 & -3.94 \\
\hline
\end{tabular}

\section{Identification of Putative Docking Sites for MsMAPKs on MsPP2Cs}

BIPSPI server was used for the prediction of docking sites. For MsMAPKs, the relation among the docking sites was analyzed by MsPP2Cs sequence characteristics, such as the position of docking sites. A list of potential docking sites was obtained through the docking approach which showed PPI among 4 
MsPP2Cs and 12 MsMAPKs. The prediction from BIPSPI was MsMAPKs and MsPP2Cs novel docking sites were obtained from protein sequence that demonstrated several potential sites. Maximum novel docking sites were predicted in NPP2C1, PP2C, MAPK, and MSK-2 (Table 2). The novel docking sites were highlighted in green colour in the models of MsMAPKs and MsPP2Cs (Figure 3).

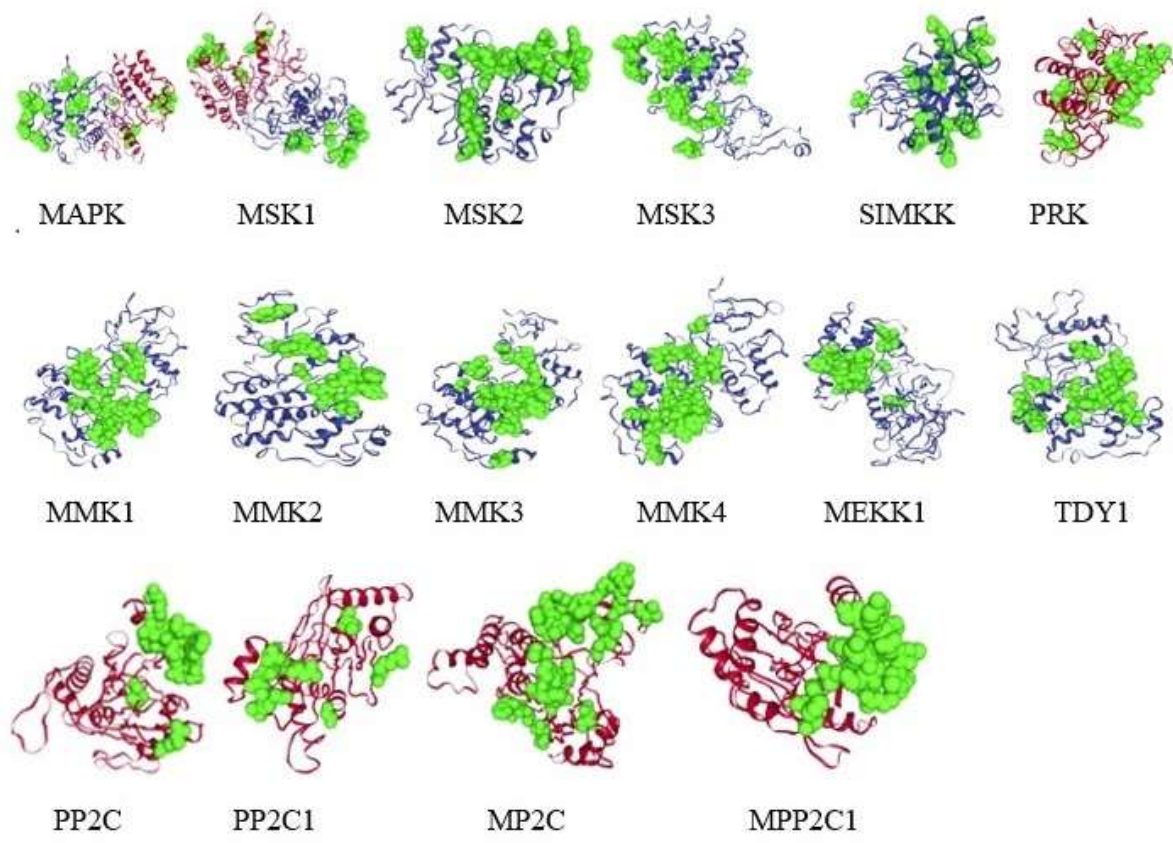

Figure 3. Visualization and prediction of binding sites obtained from 3D models of MsMAPKs and MsPP2Cc shown in green colour.

Table 2. List of binding sites obtained from BIPSPI server

\begin{tabular}{lll}
\hline $\begin{array}{l}\text { Docking } \\
\text { proteins }\end{array}$ & Docking site & Residues \\
\hline MP2C & DGHGDCRRSDG & $161,162,163,234,235,236,237,288,321,322,323$ \\
npp2c1 & FDGHGPYFDSDESR & $99,100,101,102,103,104,105,2003,226,227,375,469,472,473$ \\
PP2C1 & YDGHGGDRSDG & $90,91,92,93,94,175,176,228,262,263$ \\
PP2C & RYDGHGRSDGLWDN & $27,135,136,137,138,139,278,311,312,313,314,315,374,375$ \\
Mekk1 & IKFWMWSL & $443,444,480,481,482,501,502,503$ \\
MAPK & RERKNEWMAPEYSF & $121,143,190,193,196,231,232,233,234,235,236,250,251,252$ \\
MSK-1 & QDKRYKNRESRYR & $107,108,109,110,111,112,113,114,115,237,238,239,279$ \\
MSK-2 & LQDKRYKNREMRYS & $105,106,107,108,109,110,111,112,113,114,165,237,238,278$ \\
MSK-3 & QDKRYKNRERYES & $106,107,108,109,110,111,112,113,114,237,238,277,278$ \\
PRK & MDRDLKPSYMRS & $147,148,190,191,192,193,194,195,232,233,243,253$ \\
MMK1 & LREYTRWYRA & $99,100,101,214,217,218,219,220,221,222$ \\
MMK2 & REDYTRWYRAN & $82,83,180,196,199,200,201,202,203,204,268$ \\
MMK3 & YLREEYTRWYRA & $50,84,85,86,198,199,202,203,204,205,206,207$ \\
MMK4 & RYTRWYRA & $84,198,201,202,203,204,205,206$ \\
Simkk & RKPYMINDYWS & $201,204,205,243,244,253,254,255,258,266,267$ \\
TDY1 & RYTRWYRA & $70,188,191,192,193,194,195,196$ \\
\hline & &
\end{tabular}




\section{Comparison of MAPKs Putative Docking Sites}

Comparison of novel docking sites of MAPKs was performed. MAPK interacting protein has a particular docking region at the $\mathrm{N}$-terminus indicated the CD sites of MAPKs binding (Figure 4a). Those amino acids having positive charge found in the external region of the catalytic domains (Figure 4b) Comparison of docking sites of SIMKK with AtMMK2, AtMMK4, AtMMK5, DMEK, MEK1, MEK3 and Ste7 [28]. The potential residues of specific sub-motifs $(+++)$ are shown in yellow while red colour indicated hydrophobic sub-motifs $(-x-)$ (Figure 4c).

A

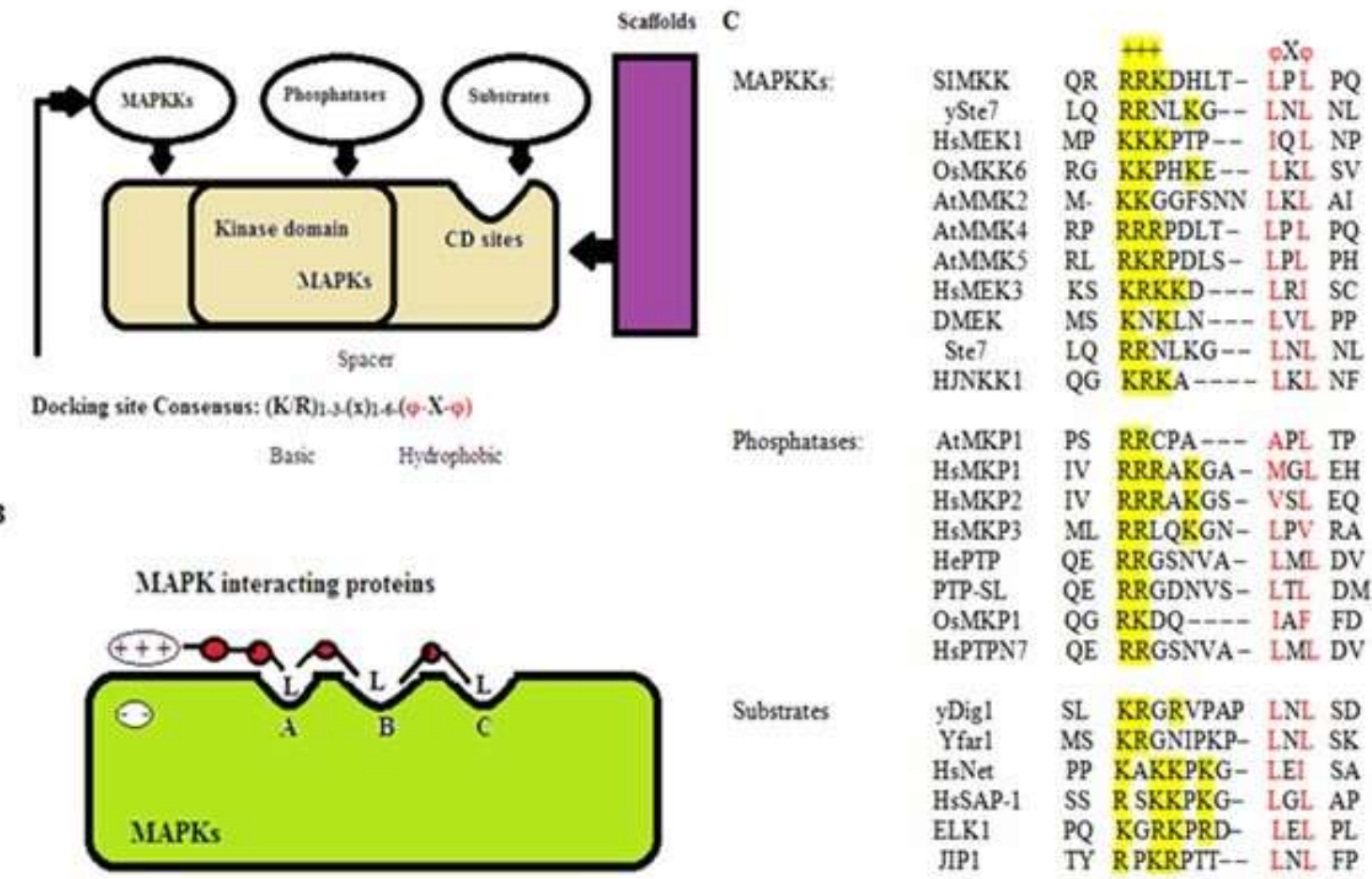

Figure 4. Interaction of MAPKs with docking sites on substrates and regulators. (a) MAPKs docking sites and different classes of MAPKs interacting proteins (b) The complex of potential proteins having interaction with MAPKs. (c) Comparison of MAPKs docking sites found in yeast, human and plants. Residues having basic sub-motifs $(+++)$ highlighted in yellow colour while hydrophobic sub-motifs $(-x-)$ shown in red colour.

\section{DISCUSSION}

MAPKs proteins are expressed mainly in roots and stems of alfalfa [29]. MsPP2Cs caused to dephosphorylate the MsMAPKs. PP2Cs act as negative regulators of MAPKs that activate in plants during stress. It is studied that MsPP2Cs (PP2Cs in M. sativa) function in negative regulation of MAPKs [30].

The MsMAPKs and MsPP2Cs three-dimensional structures give useful knowledge about the role of the molecular bases of the proteins [31]. Therefore, three-dimensional models of proteins with high-resolution are major factor which helps to understand the molecular and biochemical functions [32]. The experimental detection of protein structure was difficult, time-consuming, expensive, and unsuccessful [33]. Therefore, homology modeling becomes an effective approach that helps to reduce the gap between known protein sequence and protein structure obtained through experiments. By choosing templates and target-template sequence alignment, the Swiss model generates an automated protein structure [34]. Homology modeling is known to be the highly accurate and precise technique of computational prediction of three-dimensional models [35]. In the prediction of main residues, the potential residues that perform an essential function in PPI could be identified. Therefore, HawDock is an efficient server for the prediction of binding structures and PPI residues [19].

PPI on a large-scale have been found by experimentally in past but these laboratory techniques were difficult for new target organism. Due to this reason, in silico techniques were generated to detect proteinprotein interaction [36]. Docking study is used for prediction of PPI among MAPKs and PP2Cs which are linked together to develop highly stable complex structures with the lowest energies and greater chance of 
interaction. Therefore, docking is a requirement for enzymatic reaction and identification of the interaction of molecular protein [37].

Our findings showed that computational techniques such as docking could effectively predict PPI between MsMAPKs and MsPP2Cs which was cheaper, easy, and effective as compared to laboratory methods. Such findings recommended the linkage of PP2Cs with many other cellular processes [38]. The predicted results demonstrated that the prediction of PPIs has structural support for mutual recognition and also demonstrating the interactions among MsMAPKs with MsPP2Cs. It has been reported that PP2Cs act in the negative regulation of MAP kinases [39]. The understanding of the interaction between MAPKs and PP2Cs is very important. In the N-terminus region, MAPK interacting protein usually has sites of docking that can display MAPKs binding CD domains [40].

There are hydrophobic grooves (-X-grove) that have three side chains docking pockets at the putative docking sites of MAPKs. The CD sites combine to essential residues at the docking motif's N-terminal region. Alfalfa SIMKK has potential residues that are preserved in MAPKK, phosphatase, and transcription factor at N-terminal (Figure 4a, b, c) [28]. The area of MAPK interacting motif was recognized as PP2Cs in subgroup B [41]. PP2Cs have been recognized in another type of potential site with motifs named DEF has FXFP in plants, yeast, and human [42]. Through gene expression, MAPKs were regulated by transcription between receptor and cell response [43]. MAPKs are important protein phosphorylation components that deliver a signal for plant protection [44]. This study predicted that MsMAPKs show interaction with MsPP2Cs disclose the signaling pathway for a disease that uses the docking method.

The study on the functions of MAPKs signaling, especially their role as a negative regulator with PP2Cs is extremely limited. It has been explored that MP2C acts as a negative regulator of SAMK that is activated by stresses [45]. A large number of computational tools have been generated to accurately and precisely predict PPIs [38]. The first stage in the systematic study of the specific plant is the development of a network of proteins that not only provides knowledge related to signaling pathways but also gives an understanding of protein's functions [46]. PPIs have been discovered in Arabidopsis thaliana [47], Saccharomyces cerevisiae [48], Triticum aestivum, and Oryza sativa [49]. In A. thaliana, the signaling pathway of MAPKs has been discovered which helps in the understanding of cellular mechanisms [47]. In S. cerevisiae, PPI was discovered between 1730 proteins which revealed 7714 interacting pairs [48]. The study of PPI in wheat and rice revealed $83 \%$ interaction of proteins of rice with wheat which was important for the investigation of potential interacting proteins in wheat [49]. In B. distachyon, PPIs were predicted in 16 BdMAPKs and 86 BdPP2Cs through docking approach which revealed 96 interacting pairs [13].

\section{CONCLUSION}

For PPI prediction among 12 MsMAPKs and 4 MsPP2Cs, the computational 'Docking approach' was used in this study. Docking studies show 48 interaction pairs suggested that MsPP2Cs could engage in many cellular mechanisms. Furthermore, mostly MsPP2Cs have potential residues that are docked with MsMAPKs suggested that sites of docking perform an important role in the binding of MsPP2Cs and MsMAPKs. These findings provide knowledge about the interaction of docking proteins in the MAPKs signaling pathway. It must be linked with the identification of new MAPK substrates and regulators by in silico techniques. However, MsPP2Cs and MsMAPKs were preferred for PPIs prediction. This study suggested that protein-protein interaction among MsMAPKs and MsPP2Cs has structural support evidence.

Funding: This research received no external funding.

Acknowledgement: The authors are highly grateful to the University of Education, Lahore for providing a platform to conduct this research.

Conflicts of Interest: The authors declare no conflict of interest.

\section{REFERENCES}

1. McCarty DR, Chory J. Conservation and innovation in plant signaling pathways. Cell 2000; 103(2): 201-9.

2. Umbrasaite J, Schweighofer A, Meskiene I. Substrate analysis of Arabidopsis PP2C-type protein phosphatases. In Plant Kinases 2011; 149-61.

3. Sinha AK, Jaggi M, Raghuram B, Tuteja N. Mitogen-activated protein kinase signaling in plants under abiotic stress. Plant Signal. Behav. 2011; 6(2):196-203.

4. Colcombet J, Sözen C, Hirt H. Convergence of multiple MAP3Ks on MKK3 identifies a set of novel stress MAPK modules. Front Plant Sci. 2016; 7: 1941. 
5. Bardwell AJ, Frankson E, Bardwell L. Selectivity of docking sites in MAPK kinases. J Biol Chem. 2009; 284(19): 13165-73.

6. Sugimoto H, Kondo S, Tanaka T, Imamura C, Muramoto N, Hattori E, Ohto C. Over expression of a novel Arabidopsis PP2C isoform, AtPP2CF1, enhances plant biomass production by increasing inflorescence stem growth. J Exp Bot. 2014; 65(18): 5385-5400.

7. Li MG, Katsura K, Nomiyama H, Komaki KI, Ninomiya-Tsuji J, MatsumotoK Tamura S. Regulation of the interleukin1-induced signaling pathways by a novel member of the protein phosphatase $2 \mathrm{C}$ family (PP2C $\varepsilon$ ). J Biol Chem. 2003; 278(14): 12013-21.

8. Šamajová $\mathrm{O}$, Plíhal $\mathrm{O}$, Al-Yousif $\mathrm{M}$, Hirt $\mathrm{H}$, Šamaj J. Improvement of stress tolerance in plants by genetic manipulation of mitogen-activated protein kinases. Biotechnol Adv. 2013; 31(1): 118-28.

9. Shubchynskyy V, Boniecka J, Schweighofer A, Simulis J, Kvederaviciute K, Stumpe M, Zipfel C. Protein phosphatase AP2C1 negatively regulates basal resistance and defense responses to Pseudomonas syringae. J Exp Bot. 2017; 68(5):1169-83.

10. Braun P, Gingras AC. History of protein-protein interactions: From egg white to complex networks. Proteomics 2012; 12(10): 1478-98.

11. Tress M, de Juan D, Graña O, Gómez MJ, Gómez-Puertas P, González JM, Valencia A. Scoring docking models with evolutionary information. Proteins: Structure, Function, and Bioinformatics. 2005; 60(2): 275-80.

12. Huang YA, You ZH, Chen X, Chan K, Luo X. Sequence-based prediction of protein-protein interactions using weighted sparse representation model combined with global encoding. BMC Bioinformatics 2016; 17(1): 184 .

13. Jiang M, Niu C, Cao J, Ni DA, Chu Z. In silico-prediction of protein-protein interactions network about MAPKs and PP2Cs reveals a novel docking site variants in Brachypodium distachyon. Sci Rep. 2018; 8(1): 1-11.

14. Collins SR, Kemmeren P, Zhao XC, Greenblatt JF, Spencer F, Holstege FC, Krogan NJ. Toward a comprehensive atlas of the physical interactome of Saccharomyces cerevisiae. Mol Cell Proteomics. 2007; 6(3): 439-50.

15. Barradas-Bautista D, Rosell M, Pallara C, Fernández-Recio J. Structural Prediction of Protein-Protein Interactions by Docking: Application to Biomedical Problems. Adv Protein Chem Struct Biol.2018; 110: 203-49. Academic Press.

16. Sun T, Zhou B, Lai L, Pei J. Sequence-based prediction of protein-protein interaction using a deep-learning algorithm. BMC Bioinformatics. 2017; 18(1): 1-8.

17. Huang SY. Search strategies and evaluation in protein-protein docking: principles, advances and challenges. Drug Discov Today. 2014; 19(8): 1081-96.

18. Vakser IA. Protein-protein docking: From interaction to interactome. Biophys J. 2014; 107(8):1785-93.

19. Weng G, Wang E, Wang Z, Liu H, Zhu F, Li D, et al. HawkDock: a web server to predict and analyze the proteinprotein complex based on computational docking and MM/GBSA. Nucleic Acids Res. 2019; 47(W1): W322-30.

20. Zacharias M. Protein-protein docking with a reduced protein model accounting for side chain flexibility. Protein Sci. 2003; 12(6): 1271-82.

21. Chen T, Guestrin C. Xgboost: A scalable tree boosting system. In Proceedings of the 22nd acm sigkdd international conference on knowledge discovery and data mining 2016: 785-94.

22. Feng T, Chen F, Kang Y, Sun H, Liu H, Li D, Hou T. HawkRank: a new scoring function for protein-protein docking based on weighted energy terms. J Cheminformatics 2017; 9(1): 66.

23. Brock AK, Willmann R, Kolb D, Grefen L, Lajunen HM, Bethke G, et al. The Arabidopsis mitogen-activated protein kinase phosphatase PP2C5 affects seed germination, stomatal aperture, and abscisic acid-inducible gene expression. Plant Physiol. 2010; 153(3): 1098-1111.

24. Biasini M, Schmidt T, Bienert S, Mariani V, Studer G, Haas J, Schwede T. Open Structure: an integrated software framework for computational structural biology. Acta Crystallogr D Biol Crystallogr 2013; 69(5): 701-09.

25. Laskowski RA, MacArthur MW, Thornton JM. PROCHECK: Validation of protein-structure coordinates. International Tables for Crystallography 2006; 25(2): 722-25.

26. Premalatha D, Ravindra P, Rao LV. Homology modeling of putative thioredoxin from Helicobacetr pylori. Indian $\mathrm{J}$ Biotechnol. 2007; 6:485-89.

27. Futran AS, Link AJ, Seger R, Shvartsman SY. ERK as a model for systems biology of enzyme kinetics in cells. Curr Biol. 2013; 23(21): R972-79.

28. Kiegerl S, Cardinale F, Siligan C, Gross A, Baudouin E, Liwosz A, Meskiene I. SIMKK, a mitogen-activated protein kinase (MAPK) kinase, is a specific activator of the salt stress-induced MAPK, SIMK.Plant Cell. 2000; 12(11): 224758.

29. Ovečka M, Takáč T, Komis G, Vadovič P, Bekešová S, Doskočilová A, Meskiene I. Salt-induced subcellular kinase relocation and seedling susceptibility caused by overexpression of Medicago SIMKK in Arabidopsis. J Exp Bot. 2014; 65(9): 2335-50. 
30. Kondoh K, Nishida E. Regulation of MAP kinases by MAP kinase phosphatases. Biochim Biophys Acta Mol Cell Res.2007; 1773(8):1227-37.

31. Cavasotto CN, Phatak SS. Homology modeling in drug discovery: current trends and applications. Drug Discov Today 2009; 14(13-14): 676-83.

32. Joo K, Lee J, Lee J. Methods for accurate homology modeling by global optimization. In Homology Modeling 2011; $175-88$.

33. Floudas CA, Fung HK, McAllister SR, Mönnigmann M, Rajgaria R. Advances in protein structure prediction and de novo protein design: A review. Chem Eng Sci. 2006; 61(3): 966-88.

34. Waterhouse A, Bertoni M, Bienert S, Studer G, Tauriello G, Gumienny R, et al. SWISS-MODEL: homology modeling of protein structures and complexes. Nucleic Acids Res. 2018; 46(W1): W296-W303.

35. Muhammed MT, Aki Yalcin E. Homology modeling in drug discovery: Overview, current applications, and future perspectives. Chem Biol \& Drug Des. 2019; 93(1):12-20.

36. Ding Z, Kihara D. Computational identification of protein-protein interactions in model plant proteomes. Sci Rep. 2019; 9(1):1-13.

37. Peterson LX, Togawa Y, Esquivel-Rodriguez J, Terashi G, Christoffer C, Roy A, Kihara D. Modeling the assembly order of multimeric heteroprotein complexes. PLoS Comput Biol.2018; 14(1): e1005937.

38. Li JQ, You ZH, Li X, Ming Z, Chen X. PSPEL: in silico prediction of self-interacting proteins from amino acids sequences using ensemble learning. IEEE/ACM Trans Comput Biol Bioinf. 2017; 14(5):1165-72.

39. Martín H, Flández M, Nombela C, Molina M. Protein phosphatases in MAPK signalling: we keep learning from yeast. Mol Microbiol. 2005; 58(1): 6-16.

40. Bardwell L. Mechanisms of MAPK signalling specificity. Biochem Soc Trans. 2006; 34(5): 837-41.

41. Schweighofer A, Kazanaviciute V, Scheikl E, Teige M, Doczi R, Hirt H, Buchala A. The PP2C-type phosphatase AP2C1, which negatively regulates MPK4 and MPK6, modulates innate immunity, jasmonic acid, and ethylene levels in Arabidopsis. Plant Cell. 2007; 19(7): 2213-24.

42. Jacobs D, Glossip D, Xing H, Muslin AJ, Kornfeld K. Multiple docking sites on substrate proteins form a modular system that mediates recognition by ERK MAP kinase. Genes dev. 1999; 13(2):163-75.

43. Meier I. Composition of the plant nuclear envelope: theme and variations. J Exp Bot. 2007; 58(1): 27-34.

44. Reményi A, Good MC, Bhattacharyya RP, Lim WA. The role of docking interactions in mediating signaling input, output, and discrimination in the yeast MAPK network. Mol Cell. 2005; 20(6): 951-62.

45. Meskiene I, Baudouin E, Schweighofer A, Liwosz A, Jonak C, Rodriguez PL et al. Stress-induced protein phosphatase 2C is a negative regulator of a mitogen-activated protein kinase. J Biol Chem. 2003; 278(21): 1894552.

46. Singh R, Lee JE, Dangol S, Choi J, Yoo RH, Moon JS, Jwa NS. Protein interactome analysis of 12 mitogen activated protein kinase kinase kinase in rice using a yeast two hybrid system. Proteomics 2014; 14(1): 105-15.

47. Mingyu Z, Zhengbin Z, Shouyi C, Jinsong Z, Hongbo S. WRKY - transcription factor superfamily: structure, origin and functions. Afr. 2012; 11(32): 8051-59.

48. Cai L, Pei Z, Qin S, Zhao X. Prediction of protein-protein interactions in saccharomyces cerevisiae based on protein secondary structure. International Conference on Biomedical Engineering and Biotechnology 2012: pp.413-416.

49. Cantu D, Yang B, Ruan R, Li K, Menzo V, Fu D, Dubcovsky J. Comparative analysis of protein-protein interactions in the defense response of rice and wheat. BMC Genomics 2013; 14(1): 166.

2021 by the authors. Submitted for possible open access publication under the terms and conditions of the Creative Commons Attribution (CC BY NC) license (https://creativecommons.org/licenses/by-nc/4.0/). 